Ann. Biol. anim. Bioch. Biophys., I975, 15 (4), 697-704.

\title{
L'OVOCYTE EN PROPHASE I DE MÉIOSE : UN MODELE CYTOLOGIQUE POUR L'ÉTUDE DES GENES QUI CODENT POUR LES ARN RIBOSOMIQUES
}

\author{
A. STAHL, J. M. LuCIANI, Monique DEvictor, Michèle HaRTUNG, \\ Anne-Marie CAPODANO, C. MIRRE et D. PARDO \\ Laboratoive d'Histologie et Embryologie II. \\ Faculté de Médecine, \\ 27, boulevard Jean-Moulin, \\ 13385 Marseille Cedex 4
}

\section{RÉSUMÉ}

L'ovocyte humain au stade diplotène montre de nombreux micronucléoles associés à des régions hétérochromatiques. Parmi celles-ci, on peut identifier spécifiquement la constriction secondaire du chromosome no 9 .

L'ovocyte de la Caille constitue un matériel particulièrement favorable pour l'étude des cistrons ribosomiques. Chez cette espèce, les organisateurs nucléolaires sont situés sur des microchromosomes. Au stade pachytène, les régions hétérochromatiques des microchromosomes fusionnent en constituant des chromocentres, les segments euchromatiques restant libres. Au stade diplotène, de volumineux nucléoles se forment en relation avec les chromocentres et les segments euchromatiques des microchromosomes. L'étude ultrastructurale montre que le segment euchromatique d'un microchromosome aborde le nucléole dans une zone finement fibrillaire et pénètre à l'intérieur de celui-ci. L'hybridation in situ localise les cistrons ribosomiques, au stade pachy. tène, dans une zone limitée des microchromosomes, située à l'émergence du chromocentre. Cette localisation est confirmée par l'incorporation d'uridine tritiée.

L'ovocyte de certaines espèces est connu pour l'intensité des processus d'élaboration nucléolaire qui ont lieu au stade diplotène. Chez les Amphibiens, à ce stade, le noyau se remplit de centaines de nucléoles. Comme GaLL (I968) 1'a montré, ceci est dû à une replication sélective de l'organisateur nucléolaire pendant le stade pachytène, qui aboutit à la formation d'un $\mathrm{ADN}$ extrachromosomique. Celui-ci se disperse dans le noyau sous la forme de molécules circulaires (MILLER, I964). Chacune de ces dernières donne naissance à un nucléole. Par ailleurs, BRown et DAwID (I968) ont 
isolé, à partir du noyau de l'ovocyte du Xénope, un ADN satellite, qui a pu être localisé par McGregor (Ig68) dans 1'ADN extrachromosomique. Cet ADN satellite peut être hybridé avec les ARN ribosomiques $28 \mathrm{~S}$ et I $8 \mathrm{~S}$ dans la proportion de I8 p. Ioo de son poids (GALL, I969).

L'étude de l'ovocyte d'A cheta par LimA-DE-FARIA (I969, I973, I974) a également mis en relief des processus d'amplification des cistrons ribosomiques, qui se font dans deux volumineux chromomères du stade pachytène.

Des recherches préliminaires faites sur l'ovocyte humain à l'aide de techniques cytologiques et cytogénétiques ont permis de déceler des aspects évocateurs de processus du même ordre. (Luciani et Stahl, I97I ; STAhL et Luciani, I972 ; STAhL et al., I974, I975). Il était nécessaire, d'une part de vérifier que les observations faites sur l'ovocyte humain pouvaient s'étendre à l'ensemble des Amniotes, d'autre part de disposer d'un matériel d'obtention plus aisée et se prêtant mieux à l'expérimentation que l'ovocyte humain. A la suite de la description par LE DouARIN (I969, I973) des particularités du noyau cellulaire de la Caille, nous avons opté pour l'ovocyte de cette espèce. Ce choix s'est avéré remarquablement fécond en raison de l'association étroite chez la Caille des organisateurs nucléolaires et de l'hétérochromatine.

\section{MATÉRIEL ET MÉTHODES}

Les ovaires humains proviennent d'avortements thérapeutiques effectués à 5 mois, 5 mois I/2 et 6 mois et de prématurés décédés à 8 mois $\mathrm{I} / 2$

L'ébauche ovarienne gauche de Cailles japonaises (Coturnix coturnix japonica) âgées de 6 heures, I2 heures, 24 heures et $4^{8}$ heures après l'éclosion a été prélevée sur des animaux anesthésiés à l'éther.

Pour l'obtention d'ovocytes isolés et bien étalés, la technique de Luciasi et al. (I974) a été utilisée : les fragments d'ovaire sont fixés dans du méthanol-acide acétique (3/I). Ils sont dilacérés dans la même solution. L'isolement des cellules s'obtient par passage dans l'acide acétique à 45 p. Ioo. La suspension est étalée sur lames glacées. Ce procédé fournit des cellules germinales et somatiques séparées les unes des autres et parfaitement étalées.

Pour la microscopie photonique, les colorations suivantes ont été pratiquées : Giemsa, orcéine acétique, technique d'ARRIGH et Hsu (I97I), technique de Gagne et LABERGE (pour l'ovocyte humain seulement), réaction de la pseudoisocyanine de STERBA (I963) adaptée aux chromosomes humains selon VAGNER-CAPODANO et al. (I975). L'étude en fluorescence des préparations après coloration par l'orange d'acridine a été faite soit directement, soit après traitement des lames par la ribonucléase $\left(\mathrm{I} \mathrm{mg} / \mathrm{ml}\right.$ ) pendant 3 heures à $37^{\circ} \mathrm{C}$.

L'étude en microscopie électronique des ovocytes de Caille a été faite après fixation par le glutaraldéhyde et post-fixation à l'acide osmique. Après inclusion à l'épon, les coupes ultra-fines réalisées au couteau de diamant ont été contrastées par l'uranyle-plomb. Certains fragments ont été fixés par le glutaraldéhyde seul pour ètre traités par la technique de mise en évidence des ribonucléoprotéines par l'EDTA de BERNHARD (I969).

L'hybridation $\mathrm{rADN} / \mathrm{rARN}$ in situ a été pratiquée sur des préparations d'ovocytes humains et sur des préparations d'ovocytes de Caille. Les ARN ribosomiques marqués ont été préparés à partir de cellules Hela en utilisant la technique d'Ascione et Arlinghaus (i97o). De l'uridine tritiée (activité spécifique : $20 \mathrm{Ci} / \mathrm{mM}-\mathrm{CEA}$ ) a été introduite dans les cultures à raison d'i $\mathrm{mCi}$ par boîte Falcon de $75 \mathrm{~cm}^{2}$. Isolés sur gradient de saccharose, les ARN ont été purifiés sur colonne de Sephadex G-25. L'hybridation a été faite selon la technique de GALL et PARdue (I969). Pour l'autohistoradiographie, les lames ont été recouvertes d'une émulsion Kodak NTB 2 ou Ilford K2 et exposés 3-6 semaines. Dans les premières séries que nous avons faites, seules les préparations d'ovocyte de Caille ont donné des résultats clairement interprétables.

L'incorporation d'uridine tritiée (activité spécifique : $10 \mathrm{Ci} / \mathrm{mM}-\mathrm{CEA}$ ) a été réalisée en plaçant des fragments d'ovaire de Cailles âgées de 12 heures dans un milieu constitué de sérum de veau et de solution de Hanks $(2: 8)$ contenant de l'uridine- ${ }^{3} \mathrm{H}$, à raison de $0,1 \mathrm{mCi}$ 
par $\mathrm{ml}$ de milieu. I.a duréc de l'incubation a été de 5 , 15 et 60 minutes. Elle a été suivie par un lavage dans le même milieu contenant de l'uridine non radioactive $(\mathrm{Img} / \mathrm{ml})$. Les préparations cytologiques ont été recouvertes d'une émulsion Ilford $K_{2}$ et exposées 3-4 semaines.

OBSERVATIONS

\section{A. - Ovocyte humain}

Au stade diplotène, en plus de 2 ou 3 nucléoles principaux, on observe l'apparition de micronucléoles, au nombre de I5 à 25. Ils contiennent de l'ARN comme les nucléoles principaux, car ils ne sont plus colorables après traitement des préparations par la ribonucléase.

L'étude des préparations colorées par la technique d'ARRIGHI et Hst montre que les micronucléoles sont situés au contact de régions hétérochromatiques. Il s'agit d'une part de l'hétérochromatine juxta-centromérique de chromosomes de grande taille, d'autre part de constrictions secondaires situées vraisemblablement sur les chromosomes $\mathrm{n}^{\circ} \mathrm{I}, 9$ et $\mathrm{I} 6$. Après coloration des préparations par la technique de GAGNE et LABERGE, le segment hétérochromatique du chromosome no 9 est électivement coloré. On constate fréquemment l'association de matériel micronucléolaire à ce segment. Ces observations ayant déjà fait l'objet de plusieurs publications (voir en particulier Stahr et al., I975), leur description détaillée n'est pas reprise dans ce travail.

\section{B. Ovocyte de Caille}

Au stade pachytène précoce, les bivalents sont bien individualisés. Les microchromosomes se présentent, soit dispersés soit groupés en petits amas où ils ont conservé leur individualité. On constate que chaque microchromosome est constitué d'une région terminale hétérochromatique correspondant à la zone du centromère et d'un segment euchromatique. Des images de transition montrent que les microchromosomes s'accolent les uns aux autres par leur région hétérochromatique.

Dans une phase plus avancée du stade pachytène, les régions hétérochromatiques des microchromosomes ont fusionné en constituant 4 à 6 chromocentres d'où émergent en rayonnant les segments euchromatiques restés libres (fig. I). A ce stade, les nucléoles sont très réduits. Lorsqu'ils sont visibles, ils se présentent sous la forme de petites sphérules toujours accolées à un chromocentre.

Au stade diplotène, on observe l'apparition de nucléoles volumineux et basophiles. Ils se forment toujours en étroite relation avec un chromocentre; assez souvent, ils sont situés entre deux chromocentres (fig. 2). Chez les Cailles âgées de 48 heures, la nucléologenèse est devenue très intense, toujours en relation avec les chromocentres ainsi qu'avec les segments euchromatiques des microchromosomes (fig. 3). L'analyse des préparations révèle que les régions euchromatiques de certains microchromosomes pénètrent dans la masse du nucléole ou sont appliquées à sa surface (fig. 4 et 5). Ces microchromosomes ne montrent pas de chiasmas. Leur trajet est ponctué de petits renflements, leur extrémité se présentant souvent sous l'aspect 
d'une petite sphérule chromophile (fig. 5). Ces observations suggèrent que ces microchromosomes sont porteurs des organisateurs nucléolaires.

L'étude au microscope électronique des ovocytes au stade diplotène montre que les chromocentres sont accolés à la membrane nucléaire. Du côté opposé, orienté vers l'intérieur du noyau, on peut voir émerger le segment euchromatique d'un microchromosome, formé d'une structure axiale simple d'où émanent et s'étendent latéralement des fibres de désoxyribonucléoprotéines. Ce chromosome aborde le nucléole dans une région où ce dernier est constitué par une structure finement fibrillaire. $\mathrm{L}_{\mathbf{a}}$ technique à l'EDTA de BERNHARD confirme la présence d'un constituant chromatinien à l'intérieur du nucléole. On ne voit jamais ressortir du nucléole un microchromosome qui y a pénétré, ce qui suggère qu'une grande étendue de sa partie euchromatique se développe entièrement dans le volume nucléolaire. Souvent, le nucléole est presque complètement accolé au chromocentre et seule une structure axiale visible dans l'hétérochromatine de ce dernier indique qu'un microchromosome s'étend dans le nucléole. Dans ce cas, il faut admettre que la totalité de l'euchromatine est intriquée avec les ribonucléoprotéines nucléolaires (fig. 6 et 7 ).

Les segments de microchromosomes qui entourent le nucléole en longeant sa surface contractent également des rapports avec liti. De leur région axiale se détachent des fibres de désoxyribonucléoprotéines, qui se dirigent vers le bord du nucléole et pénètrent dans sa masse.

La technique de l'hybridation in situ, au stade pachytène, localise les gènes qui codent pour les ARN ribosomiques à la périphérie des chromocentres (fig. 8). La radioactivité est limitée à une région étroite des microchromosomes, située exactement à l'émergence du chromocentre. Au stade diplotène, les résultats ont été différents selon les séries. Dans certains cas, on retrouve la même localisation, à la périphérie des chromocentres (fig. 9). Dans d'autres cas, la radioactivité s'étend au nucléole lui-même. Ce point nécessite de nouvelles recherches, actuellement en cours de réalisation.

Après incorporation d'uridine tritiée, les résultats diffèrent d'une part selon la durée de l'incorporation, d'autre part selon le stade étudié. Les résultats les plus nets s'observent après une incorporation de 15 minutes. On constate, au stade pachytène, l'existence d'une radioactivité à la périphérie des chromocentres, dont le contour est cerné de grains d'argent (fig. IO). La radioactivité est réduite dans le reste du noyau. Au stade diplotène, on retrouve la même radioactivité à proximité des chromocentres, mais en outre de nombreux grains d'argent jalonnent le trajet des macrochromosomes (fig. II).

\section{DISCUSSION}

Les observations faites sur l'ovocyte de Caille montrent que, chez cette espèce, les organisateurs nucléolaires sont situés sur les microchromosomes, comme Comrngs et Matroccia (I970) 1'avaient déjà noté dans les cellules somatiques. On peut toutefois se demander si tous les microchromosomes sont porteurs de cistrons ribosomiques. Sur nos préparations, les microchromosomes qui sont en relation avec le nucléole ont un aspect particulier : dépourvus de chiasmas, ils sont ponctués de renflements, 
avec une sphérule terminale. Les autres microchromosomes présentent au stade diplotène des chiasmas comme les macrochromosomes. Conrngs et MatToccia ont démontré la réplication tardive de 1'ADN des microchromosomes, ce qui est une propriété générale de 1'hétérochromatine. Ils ont pu isoler, à partir des noyaux de la Caille, un ADN satellite formé de séquences répétitives et contenant $55 \mathrm{p}$. Ioo de bases GC. BROWN et JONES (I972) ont obtenu par transcription à partir de l'ADN satellite de Caille un ARN complémentaire radioactif, qu'ils ont utilisé pour localiser cet ADN par hybridation in situ. Ils ont ainsi montré qu'il est situé dans les microchromosomes. Nos observations sur l'ovocyte au stade pachytène indiquent clairement que les microchromosomes ne sont pas hétérochromatiques sur toute leur longueur, contrairement à une opinion généralement répandue. Au stade pachytène, les microchromosomes sont bien plus longs que dans une métaphase somatique; de ce fait, leur analyse est plus aisée. On constate alors que l'hétérochromatine se trouve localisée à une extrémité des microchromosomes, dans une zone qui correspond vraisemblablement au centromère. Nous avons pu montrer que les régions hétérochromatiques des microchromosomes fusionnent au pachytène pour constituer des chromocentres d'où émergent les segments euchromatiques. A ce stade, les nucléoles sont ou absents ou réduits à une très petite sphérule accolée à un chromocentre. Les observations morphologiques suggèrent qu'il n'y a pas de synthèse nucléolaire au pachytène. Toutefois, après incorporation d'uridine tritiée pendant $I_{5}$ minutes, une radioactivité se manifeste à la périphérie des chromocentres, dans la région où précisément l'hybridation in situ localise les cistrons ribosomiques présents sur les microchromosomes. Ceci conduit à admettre que la synthèse des $A R N$ ribosomiques, si évidente au stade diplotène, débute au pachytène mais ne se traduit pas encore par l'accumulation d'un matériel visible en microscopie optique.

Au stade diplotène, la nucléologenèse est très intense et se manifeste par la formation de plusieurs nucléoles très volumineux et basophiles. Ils apparaissent et se développent au contact d'un chromocentre et en relation évidente avec le segment euchromatique de plusieurs microchromosomes. L'incorporation d'uridine tritiée se traduit par une radioactivité dans la zone de formation des nucléoles; en outre elle s'étend le long des macrochromosomes, ce qui correspond sans doute à la synthèse d'ARN messager. L'hybridation in situ localise les gènes qui codent pour les ARN ribosomiques soit à la périphérie seulement des chromocentres comme au stade pachytène, soit également dans le nucléole, sans qu'il soit possible d'expliquer cette différence d'une série à l'autre. Dans les cellules somatiques de l'ébauche ovarienne de la Caille, 1'hybridation in situ localise les cistrons ribosomiques dans les nucléoles situés à la périphérie de l'hétérochromatine ; cette dernière n'est jamais radioactive (STAHL et al., I975). Ces constatations sont en accord avec celles qui ont été faites par CUDENNEC (I974) après incorporation d'uridine tritiée dans les noyaux de cellules hépatiques de la Caille.

L'étude ultrastructurale du stade diplotène apporte des précisions du plus grand intérêt sur les relations entre les microchromosomes et le nucléole. Elle confirme que les régions hétérochromatiques des microchromosomes s'associent et apparemment fusionnent en formant un chromocentre. De ce dernier émanent les segments euchromatiques orientés vers l'intérieur du noyau. Parmi eux, certains contractent avec le nucléole des rapports très particuliers. Il n'est pas rare de voir un chromosome formé par une structure axiale simple et des fibres chromatiniennes latérales aborder le 
nucléole et pénétret dans sa masse. Cette pénétration se fait toujours dans une zone d'aspect caractéristique, finement fibrillaire et d'une faible densité aux électrons. Il est vraisemblable qu'il s'agit d'un microchromosome porteur de cistrons ribosomiques et dont une grande partie est englobée dans le matériel nucléolaire qu'il a contribué à former. Comme il ne semble pas traverser le nucléole, car on ne le voit jamais ressortir à un point quelconque de sa surface, on peut admettre que les cistrons ribosomiques s'étendent sur la majeure partie du segment euchromatique : ils seraient done fortement redondants. On observe également des microchromosomes qui longent la surface du nucléole. Des fibres latérales de désoxyribontucléoprotéines se détachent de leur région axiale et s'étendent jusqu'au nucléole dans lequel elles paraissent pénétrer. Ceci suggère que des cistrons ribosomiques se trouvent également sur des microchromosomes qui ne sont pas englobés dans le nucléole et qui sont par ailleurs porteurs de gènes d'une nature différente.

Il ressort de ces observations que l'ovocyte aux stade pachytène et surtout diplotène constitue un matériel exceptionnellement favorable pour l'étude de la localisation et du comportement des cistrons ribosomiques. Les chromosomes sont à la fois analysables par les techniques morphologiques et génétiquement actifs. C'est un net avantage par rapport aux chromosomes métaphasiques des cellules somatiques, dans lesquels la transcription est momentanément suspendue.

Dans l'ovocyte humain, les organisateurs nucléolaires sont représentés par des régions spécialisées de nombreux chromosomes qui sont par ailleurs porteurs de gènes mendéliens. La dispersion de ces régions rend leur étude difficile à l'échelle ultrastructurale. Chez la Caille, des chromosomes entiers sont spécialisés dans la fonction d'organisation nucléolaire. En raison de cette particularité, jointe à la commodité de l'expérimentation, l'ovocyte de cette espèce peut constituer un excellent modèle cytologique pour l'étude des cistrons ribosomiques.

Colloque D. G. R. S. T., Biologie de la Procréation, Paris, 7-8 mars 1975.

\section{REMERCIEMENTS}

Ce travail a bénéficié de l'aide du C. N. R. S., Équipe de recherche associée no 397 et de la D. G. R. S. T., contrat no 72-70031 (Aide à la recherche no 74-7-052I).

\section{SUMMARY}

OOCYTE AT PROPHASE I OF MEIOSIS :

A CYTOLOGICAL MODEL, FOR STUDY OF GENES CODING RIBOSOMAL, ARN

The human oocyte at diplotene stage shows numerous micronucleoli associated with heterochromatic regions. Among them, the secondary constriction of the chromosome 9 can be easily identified.

The quail oocyte represents a good material for the study of the ribosomal cistrons. In this species, the nucleolar organizers are located on microchromosomes. At pachytene stage 
the heterochromatic regions of the microchromosomes fuse forming chromocentres, the euchromatic parts remain free. At diplotene stage, voluminous nucleoli appear in relation with the chromocentres and the microchromosomes euchromatic segments. The ultrastructural study shows that the euchromatic segment of a microchromosome reaches and penetrates the nucleolus in a fibrillar region. At pachytene stage, the in situ hybridization technique locates the ribosomal cistrons in a region of the microchromosomes localized at the emergence of the chromocentre. This localization is also confirmed by labelling with ${ }^{3} \mathrm{H}-\mathrm{t}$ Tridine.

\section{RÉFÉRENCES BIBLIOGRAPHIQUES}

Arrighi F. E., Hsu T. C., I97I. Localization of heterochromatin in human chromosomes. Cytogenetics, 10, 8I-86.

Ascione R., Arlinghaus R. B., I970. Characterization and cell free activity of polyribosomes isolated from baby hamster Kidney cells. Bioch. Biophys. Acta, 204, 478-488.

BERNhaRd W., 1969. A new staining procedure for electron microscopical cytology. J. Ultrastruct. Res., 27, 250-265.

Brown D. D., DAwid I. B., I968. Specific gene amplification in oocytes. Science, 160, 272-280.

Brown J. E, Jones K. W., I972. Localization of satellite DNA in the microchromosomes of the Japanese Quail by in situ hybridization. Chromosoma, Berlin, 38, 313-318.

Comings D. li., Mattoccia E., i97o. Studies of microchromosomes and a G-C rich IDNA satellite in the Cuail. Chromosoma, Berlin, 30, 202-2I 4.

Cudennec C. Le nucleole Feulgen-positif de la Caille japonaise (Coturnix coturnix japonica). Thèse de Doctorat de Spécialité, Nantes, 1974 .

GAGNE R., LABERGE C., I972. Specific cytological recognition of the heterochromatic segment of number 9 chromosome in man. Exptl. Cell Res., 73, 239-242.

Gall J. G., I968. Differential synthesis on the genes for ribosonal RNA during amphibian oogenesis. Proc. Nat. Acad. Sci, U. S. A,, 60, 553-560.

GaLl J. G., I969. The genes for ribosomal RNA during Oogenesis. Genetics, 61, I2I-132.

Gall J. G., Pardue M. L., I969. liormation and detection of RNA-DNA hybrid molecules in cytological preparations. Proc. Nat. Acad. Sci. U. S. A., 63, 378-383.

Le Douarin N., r969. l'articularités du noyau interphasique chez la Caille japonaise (Coturnix coturnix japonica). Bull. Biol. France Belgique, 103, 435-4.52.

Le: Douarin N., 1973. A lieulgen-positive nucleolus. Exp. Cell Res., ry, 459-468.

I.IMA DE FARIA A. DNA replication and gene amplification in heterochromatin. In Handbook of Molecular Cytology (ed. LimA de FARIA), 277-325, Anlsterdam-London ; North Holland, 1969.

Lima De Faria A., Jaworska H., Gustafsson T,, I973. Release of amplified ribosomal DNA from the chromomeres of Acheta. Proc. Nat. Acad. Sci. L. S. A., 70, 80-83.

IImA DE FARIA A., I974. The molecular organization of the chromomeres of Acheta involved in ribosomal DNA amplification. Cold Spring Harbor Symposia on quantitative Biology, vol. XXXVIII, 559-57I.

Lcciani J. M., Stahl A., i97I. Rapports des nucléoles avec les chromosomes méiotiques de l'ovocyte fotal humain. C.R. Ac. Sci. Paris, I, 273, 52 I-524.

Luciani J. M., Devictor-Vuillet M., Gagne R., Stahl A., I974. An air-drying method for first meiotic prophase preparations from mammalian ovaries. J. Reprod. Fert, 36, 409-4I I.

McGregor H. C., I968. Nucleolar INA in oocytes of Xenopus laevis. J. Cell. Sci., 3, 437-444.

Miller O. I., I964. Extrachromosomal nucleolar DNA in amphibian oocytes. J. Cell Biol., $23,60 \mathrm{~A}$.

Stahl A., Luciani J. M., 1972. Nucleoli and chromosomes : their relationships during the meiotic prophase of the human fetal oocyte. Humangenctik, 14, 269-284.

Stahl A., I.uciani J. M., Devictor-Vuiflet M., ig73. Ítude chromosomique de la méiose. Colloque sur les accidents chromosomiques de la reproduction (I.N.S.L.R.M.), 197-2I8, 1'aris, I2-I4 septembre.

Stail A., Luciani J. M., Devictor M., Capodano A. M., Gagne R., I975. Constitutive Heterochromatin and Micronucleoli in the Human Oocyte at the diplotene stage. Humangenetik, 26, 3I5-327.

Staill A., l'ardo D., Mrrre C., Devictor-Vlithet M., Vagner-Capodano A. M., i975. La localisation des organisateurs nucléolaires dans les noyaux somatiques de l'ébauche ovarienne de la Caille japonaise (Coturnix coturnix japonica). C. R. Acad. Sci., D, 280, 1095-1098.

Sterba G., ig63. Ankündigung eines neuen histochenischen IDNS-Nachweises mit hoher, die Feulgenreaktion übertreffender Empfindlichkeit. Acta Biol. Med. Germ., 10, 694-700.

Vagner-Caponano A. M., Delgrossi M. H., Stalil A, 1975. L'intérêt de la pseudoisocyanine dans l'étude des chromosomes humains. Ann. Biol. anim. Bioch. Biophys., 15. 


\section{PIANCHE I}

FIG. I

Ovocyte de Caille âgée de 6 heures au stade pachytène

Les régions hétérochromatiques des microchromosomes ont fusionné en constituant des chromocentres. Les segments euchromatiques des microchromosomes restent libres et rayonnent à partir de la périphérie des chromocentres. On ne voit pas de nucléole. Coloration : Giemsa.

FIG. 2 à 5

Ovocyte de Caille au stade diplotène

FIG. 2. - Caille âgée de I2 heures. Les chromosomes sont despiralisés et montrent des chiasmas. Deux nucléoles sont visibles l'un au contact d'un chromocentre, l'autre situé entre deux chromocentres.

FIG. 3. - Caille âgée de 48 heures. De nombreux et volumineux nucléoles sont formés, au contact à la fois des chromocentres et des segments euchromatiques des microchromosomes. Coloration : Giemsa.

FIG. 4 et 5. - Détails d'ovocytes au stade diplotène, chez des Cailles âgées de I2 heures : les nucléoles sont au contact de chromocentres d'où émanent les segments euchromatiques des microchromosomes, ponctués de renflements intercalaires et terminaux. Ces microchromosomes pénètrent à l'intérieur du nucléole ou s'appliquent à sa surface. Coloration : Giemsa. 

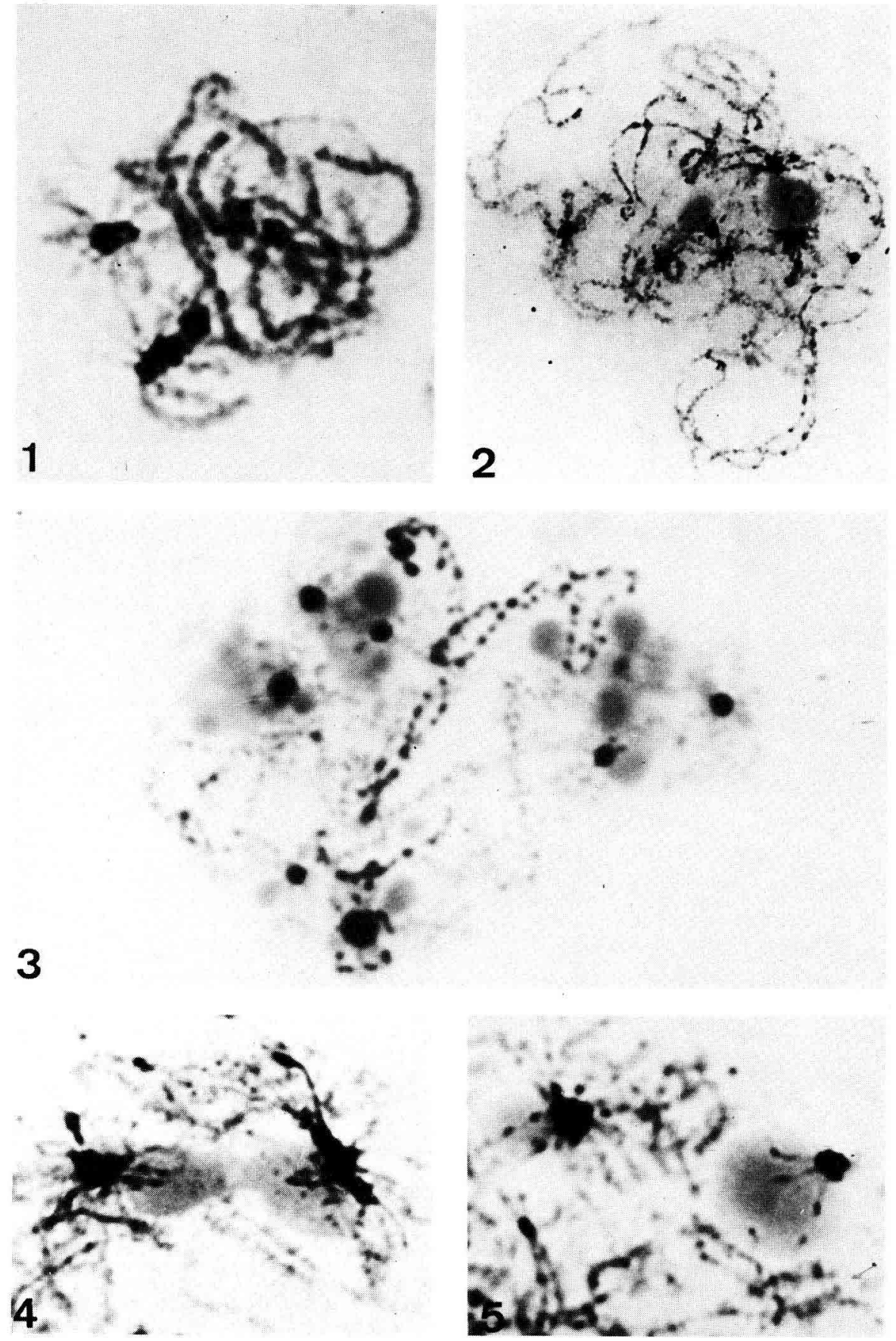

STAHL, LUCIANI, DE VICTOR et coll. 


\section{PLANCHE II}

FIG. 6

Ovocyte de Caille au stade diplotène. L'axe d'un microchromosome, issu du chromocentre, aborde le nucléole dans une zone finement fibrillaire. $\times 9.500$.

\section{FIG. 7}

Noyau d'ovocyte de Caille au stade diplotène. Détail de la zone de pénétration du microchromosome dans le nucléole. $\times 63.000$. 


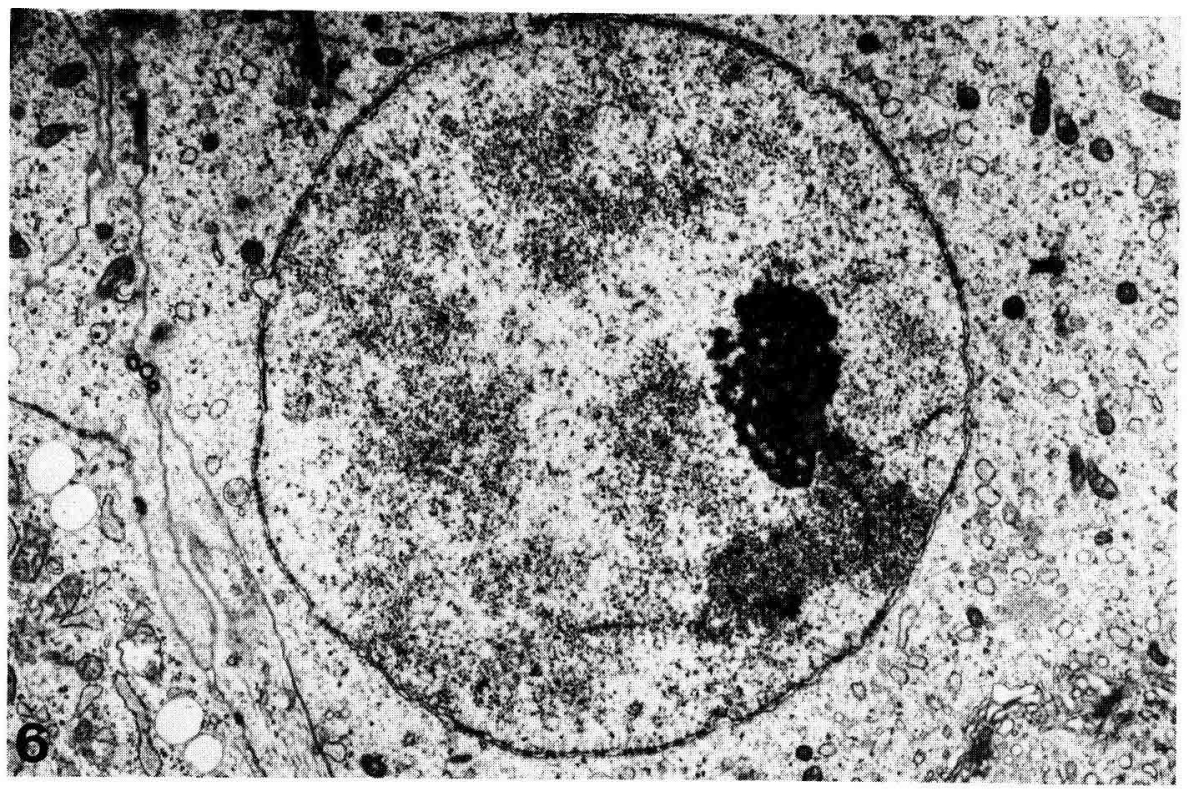

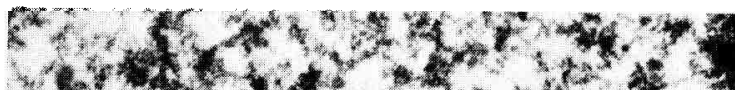
costand 10 ans $x, 1,0$

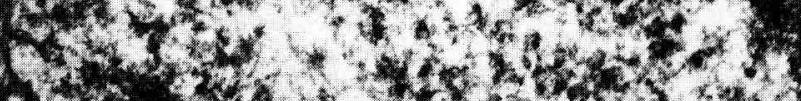

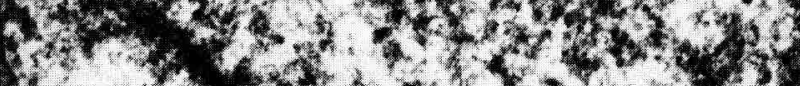

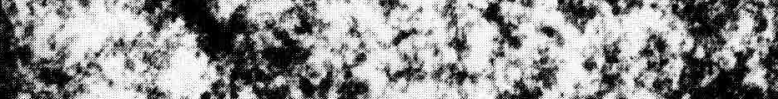

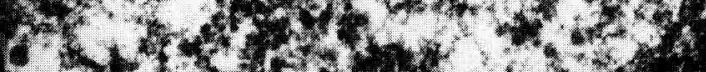

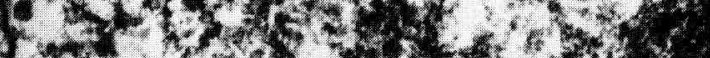

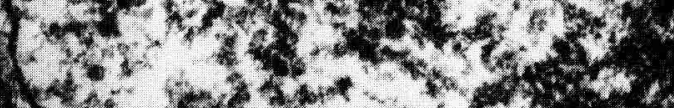

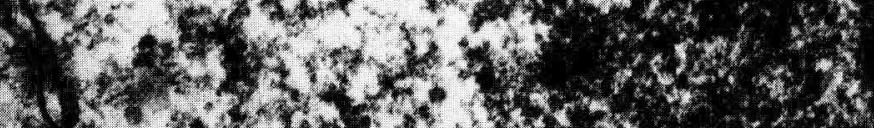
6.

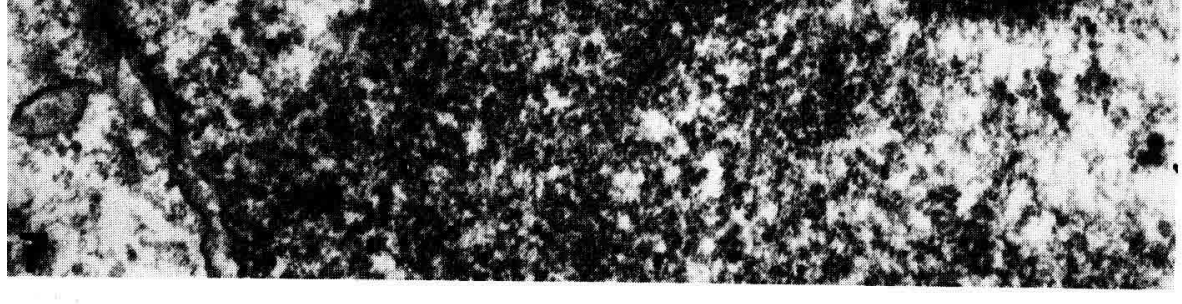

STAHL, LUCIANI, DE VICTOR et coll. 


\title{
PLANCHE III
}

\author{
FIG. 8 et 9
}

Hybridation in situ réalisée avec des ARN $28 \mathrm{~S}$ et $18 \mathrm{~S}$ marqués au tritium selon la technique de Gall et Pardue. Exposition : 1 mois.

- Fig. 8 : stade pachytène d'un ovocyte de Caille àgée de 12 heures. La radioactivité est localisée à la périphérie de 3 chromocentres, la situation des grains d'argent étant particulièrement nette autour du chromocentre le plus volumineux (flèche).

- Fig. 9 : Stade diplotène d'un ovocyte de Caille âgée de 12 heures. La radioactivité est également localisée à la périphérie des chromocentres, mais ces derniers sont plus nombreux que sur la figure 8 .

\section{FIG. 10}

Autohistoradiographie après incorporation d'uridine tritiée pendant 15 minutes dans un noyau d'ovocyte de Caille au stade pachytène. Exposition : 3 semaines. On observe la présence de grains d'argent d'une part à la périphérie des chromocentres, d'autre part le long de certains bivalents.

\section{FIG. 11}

Autohistoradiographie après incorporation d'uridine tritiée pendant 1 heure dans un noyau d'ovocyte de Caille au stade diplotène. Exposition : un mois. Les grains d'argent sont particulièrement nombreux dans la région de formation du nucléole (flèche). Microphotographie faite en contraste de phase. 

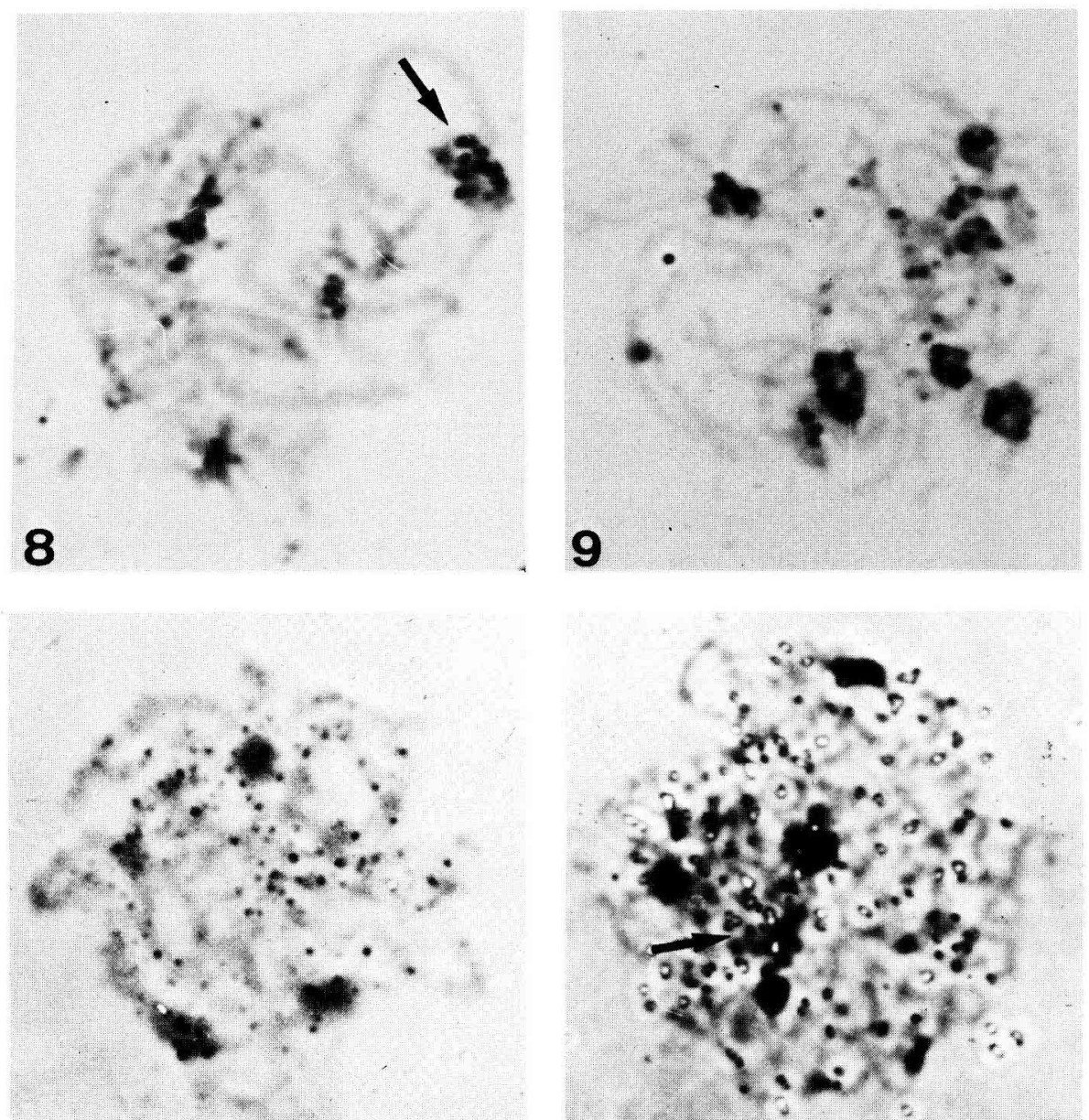

10

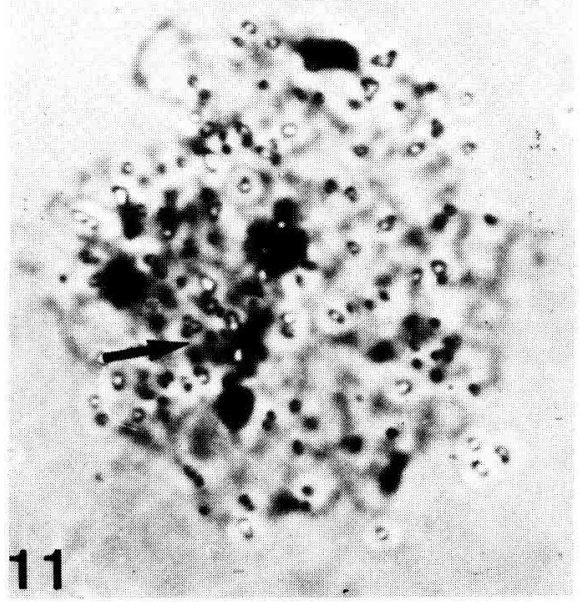

STAHL, LUCIANI, DE VICTOR et coll. 